\title{
Automatic classification of brain magnetic resonance images with hypercolumn deep features and machine learning
}

KEMAL AKYOL ( $\sim$ kemalakyol48@gmail.com)

Kastamonu Universitesi https://orcid.org/0000-0002-2272-5243

\section{Research Article}

Keywords: Brain magnetic resonance imaging, keypoint detection, hypercolumn deep features, deep learning

Posted Date: February 17th, 2022

DOI: https://doi.org/10.21203/rs.3.rs-1072357/v1

License: (ㅇ (i) This work is licensed under a Creative Commons Attribution 4.0 International License.

Read Full License 


\section{Abstract}

Brain tumours are life-threatening and their early detection is very important in a patient's life. At the present time, magnetic resonance imaging is one of the methods used for detecting brain tumours. Expert decision support systems serve specialist physicians to make more accurate diagnoses by minimizing the errors arising from their subjective opinions in real clinical settings. The model proposed in this study detects important keypoints and then extracts hypercolumn deep features of these keypoints from some convolutional layers of VGG16. Finally, Random Forest and Logistic Regression classifiers are fed with a set of these features. Random Forest classifier offered the best performance with $94.84 \%$ accuracy, $95.48 \%$ sensitivity, $4.52 \%$ false negative rate, $94.19 \%$ specificity, and $94.35 \%$ precision in 5 -fold crossvalidation in this study. Consequently, it is thought that the proposed model could contribute to field experts by integrating it into computer-aided brain magnetic resonance imaging diagnosis systems.

\section{Introduction}

Medical imaging analysis plays a vital role in detecting abnormalities. Brain tumour is considered as one of the deadliest diseases [1]. Early detection of brain tumour helps radiologists for an effective prognosis and increases the potentiality of long-term survival [2]. Detection of whether there is a brain tumour is very important in terms of planning the treatment process. Magnetic resonance imaging (MRI) is used to detect the tumour in the brain. Usually, field specialists handle out brain tumour analysis manually. However, MRI screening by field specialist is time-consuming and open to human errors. On the other hand, automatic MRI classification by an expert system decreases the workload of neurologists and helps them make final decisions. Artificial intelligence-based systems that present efficient solutions to biomedical problems have recently attracted researchers. Coronary artery disease [3], eye diseases [4, 5], breast cancer [6-11], brain tumour [12-19], Alzheimer disease [20-27] are just a few of them.

Researchers have proposed many different approaches [15, 28-30] based on image processing and machine learning for brain tumour classification which has attracted widespread attention so far. Classical machine learning approaches use hand-craft feature extraction techniques. Focusing only on low-level or high-level features and relying on handcrafted features are the main problems in the feature extraction phase of traditional machine learning [31]. The negative effects of handcrafted features on the training of classifiers have been understood over time. In line with this reason, good feature extraction is required for successful classification and modelling.

Deep learning architectures, which are sophisticated more than artificial neural networks, provided an opportunity to build models with effective and high performance. They could be successfully applied on the detection and classification of various diseases in the medical field without the need for the feature extraction process. In this context, deep learning-based classification and segmentation studies on brain MRI are quite popular and widespread. For example, Mehrotra et al. examined the performance of five pretrained deep learning algorithms on benign and malignant brain tumour images. According to the authors' studies, the AlexNet pre-trained model provided high performance compared to others[13]. 
Deepak and Ameer performed Meningioma, Glioma, and Pituitary tumour classification by sending the deep features extracted with GoogleNet pre-trained model as input to the K-Nearest Neighbors and Support Vector Machines [16]. Mohsen et al. classified the brain MRI including normal, glioblastoma, sarcoma, and metastatic bronchogenic carcinoma tumours, using deep neural networks. In the classification step, they used new features by concatenating principal component analysis and discrete wavelet transform features [14]. Kesav and Jibukumar proposed a new architecture for brain tumour classification and tumour-type object detection using region-based convolutional neural networks. In their study, the authors first classified the Glioma and healthy tumour MRI and then identified tumour regions on the Glioma MRI [32]. Tandel et al. proposed a majority voting-based ensemble algorithm to optimize overall classification performance of five convolutional neural networks-based models and also five traditional classifier-based models [33]. Marghalani and Arif identified areas of interest, using the SURF keypoint detection algorithm in their studies. Then, they extracted the features of the areas of interest and built a visual dictionary. Lastly, they classified brain tumour, Alzheimer's disease, and normal brain images, using this visual dictionary [34]. Amin et al. proposed a new method based on fused MRI sequences for brain tumour classification. They used a 23-layer CNN model to classify the segmented images obtained by a global thresholding method [35]. Shafi et al. proposed an ensemble learning method using magnetic resonance images to classify brain tumours or neoplasms and multiple sclerosis. In their study, authors first identified the region of interest about tumour and lesion and obtained the features representing these regions. They conducted majority voting prediction with the support vector machine as the base learner [36]. Swati et al. classified multiclass brain tumours, using transfer learning and block-based fine-tuning with the 5-fold cross-validation technique. The authors examined AlexNet, VGG16, and VGG19 pre-trained models, and reported that VGG19 outperformed AlexNet and VGG16 [31]. Paul et al. classified meningioma, glioma, and pituitary tumours from brain images with fully connected and convolutional neural networks models [37]. Abiwinanda et al. applied a simple CNN architecture that includes the convolution, maximum pooling, and smoothing layers followed by fully connected hidden layer to recognize glioma, meningioma, and pituitary [38]. As can be seen, CNN-based studies, specifically transfer learning approaches are widely used in the literature.

Deep learning could perform feature extraction and classification into self-learning, but generally requires a large training dataset for learning. Deep learning application and CNN training from scratch are difficult on small medical datasets [31]. In line with this subject, the analysis of magnetic resonance imaging was carried out, using machine learning and its sub-field deep learning techniques. Moreover, this approach consists of machine learning experiments on the hypercolumn deep features extracted from MRI. First, keypoint detection is conducted on the brain MRI with Oriented FAST and rotated BRIEF (ORB) keypoint detector algorithm [39]. ORB is a computationally efficient keypoint detection algorithm that is less affected by Gaussian image noise $[39,40]$. Second, the vectors including hypercolumn deep features are obtained from five different layers of the VGG16 model by taking the positions of the obtained keypoints as reference. Lastly, the feature vectors are sent as input to the Random Forest (RF) and Logistic Regression (LR) classifiers. Therefore, the performances of the classifiers on the tumour and normal cases are discussed. In this context, the main contribution of this study is as follows: 
- Keypoints are detected on the images in the training set.

- Hypercolumn deep features are extracted from different layers, and then class labelling is performed for each feature set.

- Hypercolumn deep features are sent to RF and LR classifiers. Due to the small number of images in the dataset used in this study, the VGG16 pre-trained architecture is used.

- Finally, a comparative analysis of the different models is carried out for tumour classification.

\section{Methodology}

The main aim of this study is to propose a new model that consistently and efficiently detects normal and tumour images. Classification experiments include three scenarios within the frame of hold-out and 5-fold cross-validation techniques. Scenario A includes the VGG16 modelling results. Scenarios B and C include the results based on the integration of hypercolumn deep features and traditional classifiers. After important keypoints on the image are detected, the hypercolumn deep features are extracted from these points based on the VGG16 architecture. All experimental studies in this study were implemented in a 64bit Windows 10 operating system with Intel @1.80 GHz CPU and 8 GB RAM, and Python language with Keras 2.3.1 framework.

The general block diagram of the proposed study is shown in Figure 1. As seen in this block diagram, the class prediction is performed by sending the hypercolumn features as input to the models. RF and LR classifiers predict the target class, using the hypercolumn deep features for each keypoint. Thus, the performances of different classifiers trained on hypercolumn deep features are discussed. Differently from the studies in the literature, the proposed study performs classification for each hypercolumn deep feature obtained from keypoints on the image and lastly votes them.

\subsection{Dataset}

In this study, a public brain MRI dataset prepared by [41] was used. The dataset contains 253 images, including 155 tumours and 98 normal samples. Since the number of normal ones is less than positive samples, the data augmentation technique was applied to images in the normal class. So, the dataset was balanced with 155 tumour cases and 155 normal cases. Figure 2 presents sample images.

\subsection{VGG16 deep learning architecture}

VGG16 architecture was developed by Simonyan and Zisserman [31] based on the CNN model which consists of 138 million parameters. VGG16 won the ImageNet Large Scale Visual Recognition Challenge competition held in 2014 with an accuracy score of $92.7 \%$. The VGG16 model consists of $224 \times 224$ RGB input layers, 13 convolutions, and 3 fully connected layers.

\subsection{Hypercolumn deep features}


Hypercolumn deep features are concatenation of the vector of activation outputs obtained from certain $\mathrm{CNN}$ layers for any pixel in the image. In the CNN architecture, the output layer represents the attributes to be used in classification, and the features in the previous layers have no role in classification. With the hypercolumn deep feature extraction technique, more accurate prediction is performed using the features that represent previous layers. Hypercolumn deep features are obtained by combining intermediate features extracted from the determined layers of the CNN architecture into a single vector [42]. Many studies using this technique such as Alzheimer's disease classification [43], tumour region detection [44], brain magnetic resonance imaging classification [45] are available in the literature.

\subsection{Performance metrics}

There are two classes in the dataset as tumour (positive) and normal (negative). The confusion matrix obtained in two-class classifications includes true positive (TP), true negative (TN), false positive (FP), and false negative (FN) basic criteria. Here, the TP refers to the number of positive images that were correctly classified, while the TN refers to the number of correctly classified negative images. In addition, the FN gives the number of misclassified positive images while the FP gives the number of misclassified negative images. The performances of models are measured using different metrics such as accuracy (Acc), sensitivity (Sen), false negative rate (FNR), specificity (Spe), and precision (Pre). Sen refers to the ratio of positive samples correctly classified to all positive samples, while Spe refers to the ratio of negative samples correctly classified to all negative samples. FNR is the ratio of misclassified tumour cases to all tumour cases. Accuracy indicates the general classification performance by calculating the ratio of correctly classified samples out of all samples. Precision gives the ratio of positive samples correctly classified to all cases predicted positively. These metrics were given in between Equations 1 and 4 , respectively.

$$
\text { Sen }=\frac{T P}{T P+F N}
$$

$$
F N R=\frac{F N}{F N+T P}
$$

$$
S p e=\frac{T N}{T N+F P}
$$

$$
A C C=\frac{T N+T P}{T P+F N+T N+F P}
$$


Pre $=\quad=\frac{T P}{T P+F P}(5)$

\section{Results}

\subsection{Model training}

Before model training, firstly, data augmentation techniques were applied to some images in the negative class to eliminate the unbalanced distribution problem on the dataset and improve the model performance. Image rotation such as rotate 90 degrees right, rotate 90 degrees left, rotate 180 degrees, flip vertical, and flip horizontal were applied to images selected randomly in the negative class. Therefore, each class consists of 155 images.

In this study, machine learning experiments were carried out by utilizing hold-out and 5-fold crossvalidation techniques on the balanced dataset. The hold-out technique involves the performances of traditional classifiers on the deep features extracted from pre-trained VGG16 model based on the transfer learning approach (Scenario $A$ ) and the performances of them on the hypercolumn deep features (Scenario B). With the hold-out technique, as shown in Figure 3.a, the dataset was split into 80:20 ratios for training and testing sets, respectively. So, 248 images were reserved for the training of the models and 62 images for the testing. Table 1 summarized the sub-dataset distribution according to hold-out technique.

Table 1. Information about the train and test sets

\begin{tabular}{|lllll|}
\hline \multicolumn{2}{|l}{ Original dataset } & Train (80\%) & Test (20\%) & Total \\
\hline \multirow{2}{*}{ Classes } & Normal cases & 124 & $\mathbf{3 1}$ & 155 \\
\cline { 2 - 5 } & Tumour cases & 124 & $\mathbf{3 1}$ & 155 \\
\hline \multirow{2}{*}{ Total } & & 248 & $\mathbf{6 2}$ & 310 \\
\hline
\end{tabular}

In the last scenario (Scenario $C$ ) which is the 5 -fold cross-validation technique $(k=5)$, as shown in Figure 3.b, while $80 \%$ of the dataset, which is $k-1$ parts of the dataset for each fold, was reserved for the model training, the remaining part (20\%) was reserved for model testing. Experiments that include training and testing of the models were carried out 5-times in Scenario C in this context. In Scenarios B and C, important keypoints required for training of the models were detected on the train set utilizing the ORB detector. Figure 4 shows the keypoints detected on some sample images. Hypercolumn deep features regarding these keypoints were extracted from the \#2, \#5, \#9, \#13 and \#17 of layers in VGG16 architecture. All layers in the CNN architecture could be used to extract features, but it causes system resource problems. As a result of try-and-error experiments with combinations of different layers, these layers were selected to extract hypercolumn deep features. Feature maps were upscaled to $224 \times 224$ when extracting these features. All hypercolumn feature vectors extracted from tumour and normal images are assigned to the related class. 
Dynamically obtaining the hypercolumn feature vector with the ORB keypoint detector has a limitation for CNN models because CNN-based models get input in $m x n$ sizes which $m$ and $n$ indicate the width and height of the image or matrice, respectively. The fact that detecting different keypoints from each image is a handicap to obtain $m x n$ matrix structure. For example, 19 keypoints can be detected from any image, while 189 keypoints from another image. Here, the numbers 19 and 189 are given symbolically. In this case, $19 \times 1472$ hypercolumn deep features are extracted from the first image, while 189x1472 hypercolumn deep features are extracted from the other image. 1472 is the dimension of the feature vector formed by combining the features extracted from different layers in VGG16 for any keypoint. Therefore, a limitation is available for the feature vector, which is expected as a matrix. It is inevitable to make manipulations on the feature vector to overcome this problem. Since the input data for CNN must be a matrix in $m x n$ sizes, there must be an equal number of keypoints for each image. This problem can only be overcome by:

a) Selecting randomly $p$ keypoints from each image which has many keypoints

b) Filling the missing parts of the $m x n$ matrix with zeros

Both approaches have disadvantages. While the first one causes the loss of meaningful information, the second one causes overfitting. In the proposed approach, hypercolumn deep features extracted with reference to the keypoints detected from each image in the training set were matched with the class of the image. Thus, dynamically detecting a different number of keypoint from the images is not a problem. In other words, hypercolumn deep features are dynamically extracted and target class information is assigned for each hypercolumn deep feature. So, the proposed approach in this study overcomes the disadvantages mentioned above. After hypercolumn deep features were extracted from images in the training dataset, traditional classifier-based models were trained on these features. After the model was built, the keypoint detection on the test dataset and the extraction of hypercolumn deep features from the keypoints were carried out. Then, each of the hypercolumn deep features was classified separately, and then, taking into account the hypercolumn deep features and class matches, the image was assigned to the class with the highest number of labels with the major voting approach. Some parameters of the RF and SVM classifiers used with their default parameter settings are listed in Table 2.

Table 2. Some parameters settings of the traditional classifiers

\begin{tabular}{|ll|}
\hline Classifier & Parameters with values \\
\hline RF & n_estimators=100, criterion= 'gini', max_features='auto' \\
\hline LR & solver='Ibfgs', max_iter=100, multi_class='auto' \\
\hline
\end{tabular}

\subsection{Experimental Results}

The performances of models were tested using 3 different scenarios listed below:

a. Scenario A. Results obtained on the VGG16 deep features with hold-out technique. 
b. Scenario B. Results obtained on the hypercolumn deep features with hold-out technique.

c. Scenario C. Results obtained on the hypercolumn deep features with 5-fold cross-validation technique.

In Scenario A, the classification performances of the RF and LR models on the deep features extracted from the VGG16 architecture based on the transfer learning approach were summarized with confusion matrices in Figure 5. As can be seen in the confusion matrices, the RF misclassified 6 of 31 images in the normal class as the tumour, while LR misclassified 4 of 31 images in the tumour class as the normal. In addition, RF correctly classified 28 of 31 images with tumour while LR correctly classified 24 of them.

Scenario B presents the classification performances of RF and LR classifiers on the hypercolumn deep features extracted from the images in the test dataset. Figure 6 summarized the confusion matrices obtained by the classifiers in the experiments. As seen in here, RF has less misclassification compared to LR. Accordingly, both RF and LR misclassified 3 of 31 images in the normal class. In addition, the RF classifier correctly classified 30 of 31 images with tumour while misclassifying 1 of them. LR classifier correctly classified 28 of 31 images with tumour while misclassified 3 of them. Table 3 summarizes the performance of the models on the hypercolumn deep features and VGG16 deep features within the frame of the hold-out technique.

Table 3. Classification results for hypercolumn deep features and VGG16 deep features with hold-out technique

\begin{tabular}{|lllllll|}
\hline & Classifier & Acc & Sen & FNR & Spe & Pre \\
\hline VGG16 deep features & RF & 85.48 & 90.32 & 9.68 & 80.65 & 82.35 \\
\hline Hypercolumn deep features & RF & $\mathbf{8 2 . 8 6}$ & 77.42 & 22.58 & 87.1 & 85.71 \\
\hline & LR & 93.55 & $\mathbf{9 6 . 7 7}$ & $\mathbf{3 . 2 3}$ & $\mathbf{9 0 . 3 2}$ & $\mathbf{9 0 . 9 1}$ \\
\hline
\end{tabular}

The receiver operating characteristic (ROC) curves with AUC values given in Figure 7 show the performances of both algorithms on the hypercolumn deep features and VGG16 deep features. According to the results obtained, the performances of the classifiers on the hypercolumn deep features are quite high compared to the VGG16 deep features.

Within the frame of Scenario C, the confusion matrices obtained by RF and LR classifiers for each fold were presented in Figure 8. As can be seen in the confusion matrices between fold 1 and fold 5 , the RF classifier showed better performance than LR in the proposed approach. Table 4 summarized the performance of the models on the hypercolumn deep features within the frame of the 5 -fold crossvalidation technique. The average Acc, Sen, FNR, Spe, and Pre values of RF and LR classifiers were presented in bold in this table. The ROC curves of the models are shown in Figure 9 . The RF with 0.948 average AUC value and the LR with 0.868 average AUC value confirm these results. The AUC values of RF 
are better when compared to LR. This indicates that the RF has good classification performance. Moreover, the FNRs of the classifiers used in this study were also examined in detail. Figure 10 demonstrates the FNR values of the classifiers with the 5-fold cross-validation technique. Accordingly, the LR misclassified more tumour cases as normal than RF for each fold experiments.

Table 4. 5-fold cross validation results for hypercolumn features

\begin{tabular}{|lllllll|}
\hline Fold value & Classifier & Acc & Sen & FNR & Spe & Pre \\
\hline 1 & RF & 96.77 & 100.0 & 0.0 & 93.55 & 93.94 \\
\hline 2 & LR & 91.94 & 93.55 & 6.45 & 90.32 & 90.62 \\
\hline \multirow{2}{*}{3} & RF & 95.16 & 93.55 & 6.45 & 96.77 & 96.67 \\
\hline & LR & 83.87 & 74.19 & 25.81 & 93.55 & 92.0 \\
\hline 4 & RF & 93.55 & 93.55 & 6.45 & 93.55 & 93.55 \\
\hline \multirow{2}{*}{5} & LR & 90.32 & 90.32 & 9.68 & 90.32 & 90.32 \\
\hline \multirow{2}{*}{ Average } & RF & 95.16 & 93.55 & 6.45 & 96.77 & 96.67 \\
\hline & RR & 83.87 & 80.65 & 19.35 & 87.1 & 86.21 \\
\hline & RF & 93.55 & 96.77 & 3.23 & 90.32 & 90.91 \\
\hline
\end{tabular}

The accuracies presented by the models in the experiments carried out with three scenarios were given in Figure 11. Accordingly, the performances of the classifiers on the hypercolumn deep features in Scenarios $B$ and $C$ are better than the VGG16 deep features achieved with Scenario A. Overall results show that hypercolumn deep features improved the RF and LR accuracies to $93.55 \%$ from $85.48 \%$ and $90.32 \%$ from 82.86 when compared to VGG16 deep features within the frame of the hold-out technique. In addition, hypercolumn deep features in the 5 -fold cross-validation improved the RF accuracy to $94.84 \%$ from $93.55 \%$ when compared to hypercolumn deep features with the hold-out technique. These experiments show that the proposed approach is feasible and effective for classifying brain tumours.

\section{Discussion}

Table 5 summarizes the result of this study and other studies in the literature. As seen in this table, Toğaçar et al. [45] achieved $96.77 \%$ accuracy in their study based on the selection of important ones from hypercolumn deep features. Toğaçar et al. applied the feature selection technique with the recursive feature elimination technique on the hypercolumn features obtained with pre-trained models. They classified the hypercolumn features with the SVM classifier. But, they divided the dataset into $70 \%$ 
training and $30 \%$ test set with the hold-out technique which is called simple validation in the study. Arl and Hanbay [46] achieved $97.18 \%$ classification accuracy with the deep learning-based model on the cranial MR images. But, they used quite a few images with nine patients and seven patients for training and testing, respectively with hold-out technique in the study. Different from the study of Toğaçar et al. [45], this study proposes a novel approach including keypoint detection, hypercolumn deep features, and machine learning experiments validated with the 5 -fold cross-validation technique to classify the brain MRI. The model proposed in this study showed very successful classification accuracy with $94.84 \%$.

Table 5

Comparison of proposed model and related studies

\begin{tabular}{|c|c|c|c|c|}
\hline Study & Method & $\begin{array}{l}\text { Evaluation } \\
\text { method }\end{array}$ & $\begin{array}{l}\text { \# of } \\
\text { class }\end{array}$ & $\begin{array}{l}\text { Acc } \\
(\%)\end{array}$ \\
\hline $\begin{array}{l}\text { Arı and Hanbay } \\
{[46]}\end{array}$ & $\begin{array}{l}\text { Deep learning, the extreme learning machine } \\
\text { local receptive fields }\end{array}$ & Hold-out & 2 & 97.18 \\
\hline \multirow{2}{*}{$\begin{array}{l}\text { Toğaçar et al. } \\
\text { [45] }\end{array}$} & Hyper column technique with & \multirow[t]{2}{*}{ Hold-out } & \multirow[t]{2}{*}{2} & \multirow[t]{2}{*}{96.77} \\
\hline & $\begin{array}{l}\text { convolutional neural network and feature } \\
\text { selection method }\end{array}$ & & & \\
\hline $\begin{array}{l}\text { Deepak and } \\
\text { Ameer [16] }\end{array}$ & Deep CNN-SVM & $\begin{array}{l}5 \text {-fold cross- } \\
\text { validation }\end{array}$ & 3 & 97.1 \\
\hline Swati et al. [31] & VGG-19 & $\begin{array}{l}5 \text {-fold cross- } \\
\text { validation }\end{array}$ & 3 & 94.82 \\
\hline Paul et al. [37] & Deep learning & $\begin{array}{l}5 \text {-fold cross } \\
\text { validation }\end{array}$ & 3 & 91.43 \\
\hline $\begin{array}{l}\text { Abiwinanda et } \\
\text { al. [38] }\end{array}$ & CNN + Hyper-parameters optimization & Hold-out & 3 & 84.19 \\
\hline \multirow{2}{*}{$\begin{array}{l}\text { The proposed } \\
\text { study }\end{array}$} & \multirow{2}{*}{$\begin{array}{l}\text { Hyper-column deep features and random } \\
\text { forest }\end{array}$} & Hold-out & \multirow[t]{2}{*}{2} & 93.55 \\
\hline & & $\begin{array}{l}5 \text {-fold cross- } \\
\text { validation }\end{array}$ & & 94.84 \\
\hline
\end{tabular}

\section{Conclusion}

Detection of brain tumour is very important in terms of the fast and planned treatment process and increasing the survival rate of the patient. In this study, a novel model that classifies normal and brain tumour cases was proposed. This model extracts hypercolumn features from specific layers of the VGG16 deep learning architecture for the images and classifies them with the RF classifier. Major voting technique reaches the final decision regarding the classifications for each hypercolumn deep feature vector dynamically extracted from each image. The performance of the proposed model was validated with hold-out and 5-fold cross-validation techniques. Handcrafted features are not used and minimum 
pre-processing is required in the experiments in the proposed study. The proposed approach offered the best performance with $94.84 \%$ accuracy, $95.48 \%$ sensitivity, $94.19 \%$ specificity, and $94.35 \%$ precision on the hypercolumn deep features. The proposed model could play an important role in the development of expert systems to be used in real clinical environments, taking into account its high classification accuracy. Such an expert system to be developed using a low-cost system based on deep learning architecture will help specialist physicians to make a more accurate diagnosis by minimizing errors arising from their subjective opinions.

A model with high generalization capacity on the images obtained from different resources is planned for future work. Also, using other deep architectures that could achieve a better performance in computer vision in future studies is aimed. In addition, the studies based on the different deep learning architectures on state-of-the-art images obtained by deep dream and blurry image techniques are among the future studies.

\section{Declarations}

\section{Acknowledgement}

Author would like to thank Chakrabarty [41] to provide the public brain MRI dataset.

\section{Funding}

Not applicable

\section{Conflict of Interest}

The author declares that he has no conflict of interest.

\section{Availability of data and material}

This study uses public dataset provided by Chakrabarty [41].

\section{References}

1. Sharif, M., Amin, J., Raza, M., Yasmin, M., \& Satapathy, S. C. (2020). An integrated design of particle swarm optimization (PSO) with fusion of features for detection of brain tumour. Pattern Recognition Letters, 129, 150-157. https://doi.org/10.1016/J.PATREC.2019.11.017

2. Sharif, M. I., Li, J. P., Naz, J., \& Rashid, I. (2020). A comprehensive review on multi-organs tumour detection based on machine learning. Pattern Recognition Letters, 131, 30-37. https://doi.org/10.1016/J.PATREC.2019.12.006

3. JayaSree, M., \& Koteswara Rao, L. (2020). Survey on - Identification of Coronary Artery Disease using Deep Learning. Materials Today: Proceedings. https://doi.org/10.1016/j.matpr.2020.09.526 
4. Badar, M., Haris, M., \& Fatima, A. (2020, February 1). Application of deep learning for retinal image analysis: A review. Computer Science Review. Elsevier Ireland Ltd.

https://doi.org/10.1016/j.cosrev.2019.100203

5. Perdomo, O., Rios, H., Rodríguez, F. J., Otálora, S., Meriaudeau, F., Müller, H., \& González, F. A. (2019). Classification of diabetes-related retinal diseases using a deep learning approach in optical coherence tomography. Computer Methods and Programs in Biomedicine, 178, 181-189. https://doi.org/10.1016/j.cmpb.2019.06.016

6. Liu, T., Huang, J., Liao, T., Pu, R., Liu, S., \& Peng, Y. (2021). A Hybrid Deep Learning Model for Predicting Molecular Subtypes of Human Breast Cancer Using Multimodal Data. IRBM. https://doi.org/10.1016/j.irbm.2020.12.002

7. Prasath Alias Surendhar, S., \& Vasuki, R. (2021). Breast cancers detection using deep learning algorithm. Materials Today: Proceedings. https://doi.org/10.1016/j.matpr.2020.11.600

8. Gangaputra, S., Lovato, J. F., Hubbard, L., Davis, M. D., Esser, B. A., Ambrosius, W. T., ... ACCORD Eye Research Group. (2013). COMPARISON OF STANDARDIZED CLINICAL CLASSIFICATION WITH FUNDUS PHOTOGRAPH GRADING FOR THE ASSESSMENT OF DIABETIC RETINOPATHY AND DIABETIC MACULAR EDEMA SEVERITY. Retina, 33(7), 1393-1399. https://doi.org/10.1097/IAE.0b013e318286c952

9. Pang, T., Wong, J. H. D., Ng, W. L., \& Chan, C. S. (2020, November 15). Deep learning radiomics in breast cancer with different modalities: Overview and future. Expert Systems with Applications. Elsevier Ltd. https://doi.org/10.1016/j.eswa.2020.113501

10. Singla, C., Sarangi, P. K., Sahoo, A. K., \& Singh, P. K. (2020). Deep learning enhancement on mammogram images for breast cancer detection. Materials Today: Proceedings. https://doi.org/10.1016/j.matpr.2020.10.951

11. Houssein, E. H., Emam, M. M., Ali, A. A., \& Suganthan, P. N. (2020, April 1). Deep and machine learning techniques for medical imaging-based breast cancer: A comprehensive review. Expert Systems with Applications. Elsevier Ltd. https://doi.org/10.1016/j.eswa.2020.114161

12. Saranya, C., Priya, J. G., Jayalakshmi, P., \& Pavithra, E. H. (2021). Brain tumour identification using deep learning. Materials Today: Proceedings. https://doi.org/10.1016/j.matpr.2020.11.555

13. Mehrotra, R., Ansari, M. A., Agrawal, R., \& Anand, R. S. (2020). A Transfer Learning approach for Albased classification of brain tumours. Machine Learning with Applications, 2, 100003. https://doi.org/10.1016/j.mlwa.2020.100003

14. Mohsen, H., El-Dahshan, E.-S. A., El-Horbaty, E.-S. M., \& Salem, A.-B. M. (2018). Classification using deep learning neural networks for brain tumours. Future Computing and Informatics Journal, 3(1), 68-71. https://doi.org/10.1016/j.fcij.2017.12.001

15. Saba, T., Sameh Mohamed, A., El-Affendi, M., Amin, J., \& Sharif, M. (2020). Brain tumour detection using fusion of hand crafted and deep learning features. Cognitive Systems Research, 59, 221-230. https://doi.org/10.1016/j.cogsys.2019.09.007 
16. Deepak, S., \& Ameer, P. M. (2019). Brain tumour classification using deep CNN features via transfer learning. Computers in Biology and Medicine, 111, 103345.

https://doi.org/10.1016/j.compbiomed.2019.103345

17. Hashemzehi, R., Mahdavi, S. J. S., Kheirabadi, M., \& Kamel, S. R. (2020). Detection of brain tumours from MRI images base on deep learning using hybrid model CNN and NADE. Biocybernetics and Biomedical Engineering, 40(3), 1225-1232. https://doi.org/10.1016/j.bbe.2020.06.001

18. Gupta, A., Verma, A., Kaushik, D., \& Garg, M. (2020). Applying deep learning approach for brain tumour detection. Materials Today: Proceedings. https://doi.org/10.1016/j.matpr.2020.10.063

19. Srikanth, B., \& Venkata Suryanarayana, S. (2021). Multi-Class classification of brain tumour images using data augmentation with deep neural network. Materials Today: Proceedings. https://doi.org/10.1016/j.matpr.2021.01.601

20. Li, H., Habes, M., Wolk, D. A., \& Fan, Y. (2019). A deep learning model for early prediction of Alzheimer's disease dementia based on hippocampal magnetic resonance imaging data. Alzheimer's and Dementia, 15(8), 1059-1070. https://doi.org/10.1016/j.jalz.2019.02.007

21. Abuhmed, T., El-Sappagh, S., \& Alonso, J. M. (2021). Robust hybrid deep learning models for Alzheimer's progression detection. Knowledge-Based Systems, 213, 106688. https://doi.org/10.1016/j.knosys.2020.106688

22. Bringas, S., Salomón, S., Duque, R., Lage, C., \& Montaña, J. L. (2020). Alzheimer's Disease stage identification using deep learning models. Journal of Biomedical Informatics, 109, 103514. https://doi.org/10.1016/j.jbi.2020.103514

23. Puente-Castro, A., Fernandez-Blanco, E., Pazos, A., \& Munteanu, C. R. (2020). Automatic assessment of Alzheimer's disease diagnosis based on deep learning techniques. Computers in Biology and Medicine, 120, 103764. https://doi.org/10.1016/j.compbiomed.2020.103764

24. Raza, M., Awais, M., Ellahi, W., Aslam, N., Nguyen, H. X., \& Le-Minh, H. (2019). Diagnosis and monitoring of Alzheimer's patients using classical and deep learning techniques. Expert Systems with Applications, 136, 353-364. https://doi.org/10.1016/j.eswa.2019.06.038

25. Zhou, Y., Lu, Y., \& Pei, Z. (2021). Intelligent diagnosis of Alzheimer's disease based on internet of things monitoring system and deep learning classification method. Microprocessors and Microsystems, 83, 104007. https://doi.org/10.1016/j.micpro.2021.104007

26. Bi, X., Li, S., Xiao, B., Li, Y., Wang, G., \& Ma, X. (2020). Computer aided Alzheimer's disease diagnosis by an unsupervised deep learning technology. Neurocomputing, 392, 296-304. https://doi.org/10.1016/j.neucom.2018.11.111

27. An, N., Ding, H., Yang, J., Au, R., \& Ang, T. F. A. (2020). Deep ensemble learning for Alzheimer's disease classification. Journal of Biomedical Informatics, 105, 103411. https://doi.org/10.1016/j.jbi.2020.103411

28. Ramkumar, G., Thandaiah Prabu, R., Phalguni Singh, N., \& Maheswaran, U. (2021). Experimental analysis of brain tumour detection system using Machine learning approach. Materials Today: Proceedings. https://doi.org/10.1016/j.matpr.2021.01.246 
29. V. Sabitha, J. Nayak, P. R. R. (2021). MRI brain tumour detection and classification using KPCA and KSVM. Materials Today: Proceedings. https://doi.org/10.1016/J.MATPR.2021.01.714

30. Kaplan, K., Kaya, Y., Kuncan, M., \& Ertunç, H. M. (2020). Brain tumour classification using modified local binary patterns (LBP) feature extraction methods. Medical Hypotheses, 139, 109696. https://doi.org/10.1016/j.mehy.2020.109696

31. Swati, Z. N. K., Zhao, Q., Kabir, M., Ali, F., Ali, Z., Ahmed, S., \& Lu, J. (2019). Brain tumour classification for MR images using transfer learning and fine-tuning. Computerized Medical Imaging and Graphics, 75, 34-46. https://doi.org/10.1016/J.COMPMEDIMAG.2019.05.001

32. Kesav, N., \& Jibukumar, M. G. (2021). Efficient and low complex architecture for detection and classification of Brain Tumor using RCNN with Two Channel CNN. Journal of King Saud University Computer and Information Sciences. https://doi.org/10.1016/J.JKSUCI.2021.05.008

33. Tandel, G. S., Tiwari, A., \& Kakde, O. G. (2021). Performance optimisation of deep learning models using majority voting algorithm for brain tumour classification. Computers in Biology and Medicine, 135, 104564. https://doi.org/10.1016/J.COMPBIOMED.2021.104564

34. Marghalani, B. F., \& Arif, M. (2019). Automatic Classification of Brain Tumor and Alzheimer's Disease in MRI. Procedia Computer Science, 163, 78-84. https://doi.org/10.1016/J.PROCS.2019.12.089

35. Amin, J., Sharif, M., Gul, N., Yasmin, M., \& Shad, S. A. (2020). Brain tumour classification based on DWT fusion of MRI sequences using convolutional neural network. Pattern Recognition Letters, 129, 115-122. https://doi.org/10.1016/J.PATREC.2019.11.016

36. Shafi, A. S. M., Rahman, M. B., Anwar, T., Halder, R. S., \& Kays, H. M. E. (2021). Classification of brain tumours and auto-immune disease using ensemble learning. Informatics in Medicine Unlocked, 24, 100608. https://doi.org/10.1016/J.IMU.2021.100608

37. Paul, J. S., Plassard, A. J., Landman, B. A., \& Fabbri, D. (2017). Deep learning for brain tumour classification. Medical Imaging 2017: Biomedical Applications in Molecular, Structural, and Functional Imaging, 10137, 1013710. https:// doi.org/10.1117/12.2254195

38. Abiwinanda, N., Hanif, M., Hesaputra, S. T., Handayani, A., \& Mengko, T. R. (2019). Brain Tumor Classification Using Convolutional Neural Network. IFMBE Proceedings, 68(1), 183-189. https://doi.org/10.1007/978-981-10-9035-6_33

39. Rublee, E., Rabaud, V., Konolige, K., \& Bradski, G. (2011). ORB: An efficient alternative to SIFT or SURF. In 2011 International Conference on Computer Vision (pp. 2564-2571). IEEE. https://doi.org/10.1109/ICCV.2011.6126544

40. Vinay, A., Deshpande, A. R., Pranathi, B. S., Jha, H., Murthy, K. N. B., \& Natarajan, S. (2018). Effective Descriptors based Face Recognition Technique for Robotic Surveillance Systems. Procedia Computer Science, 133, 968-975. https:// doi.org/10.1016/J.PROCS.2018.07.080

41. Chakrabarty, N. (2019). Brain MRI Images for Brain Tumor Detection I Kaggle. Retrieved March 6, 2021, from https://www.kaggle.com/navoneel/brain-mri-images-for-brain-tumourdetection/metadata 
42. Hariharan, B., Arbelaez, P., Girshick, R., \& Malik, J. (2017). Object Instance Segmentation and FineGrained Localization Using Hypercolumns. IEEE Transactions on Pattern Analysis and Machine Intelligence, 39(4), 627-639. https://doi.org/10.1109/TPAMI.2016.2578328

43. Toğaçar, M., Cömert, Z., \& Ergen, B. (2021). Enhancing of dataset using DeepDream, fuzzy color image enhancement and hypercolumn techniques to detection of the Alzheimer's disease stages by deep learning model. Neural Computing and Applications, 1-13. https://doi.org/10.1007/s00521021-05758-5

44. Wijethilake, N., Islam, M., \& Ren, H. (2020). Radiogenomics model for overall survival prediction of glioblastoma. Medical and Biological Engineering and Computing, 58(8), 1767-1777. https://doi.org/10.1007/s11517-020-02179-9

45. Toğaçar, M., Cömert, Z., \& Ergen, B. (2020). Classification of brain MRI using hyper column technique with convolutional neural network and feature selection method. Expert Systems with Applications, 149, 113274. https://doi.org/10.1016/j.eswa.2020.113274

46. Ari, A., \& Hanbay, D. (n.d.). Deep learning based brain tumour classification and detection system. https://doi.org/10.3906/elk-1801-8

\section{Figures}

\section{Figure 1}

General block diagram of the proposed model. 
a)
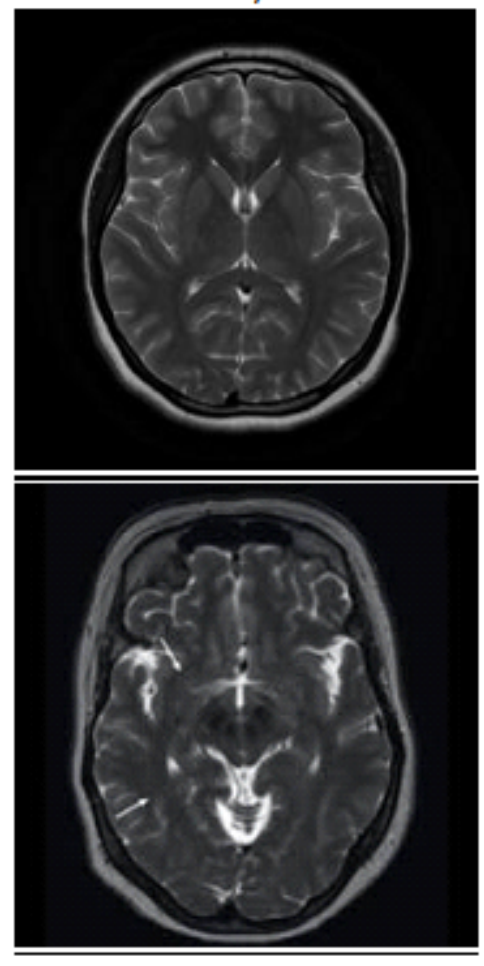

b)
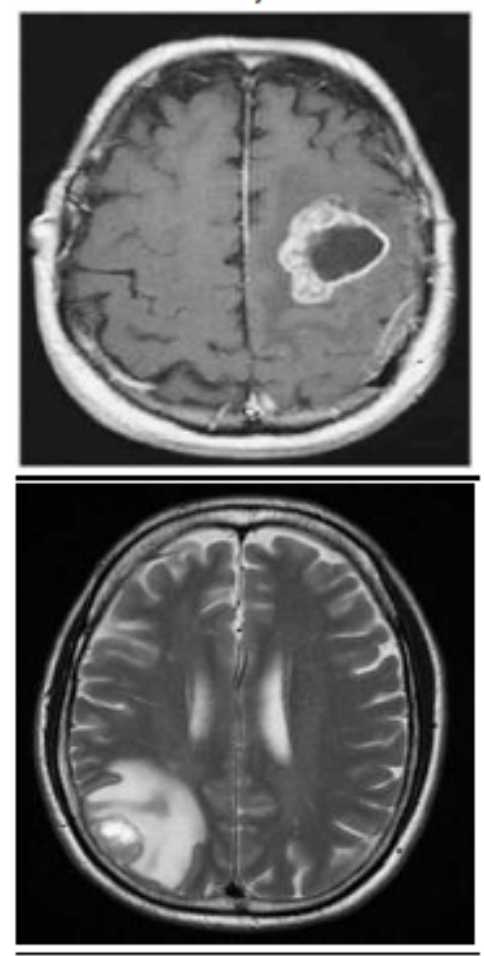

Figure 2

Samples from each class; From top to bottom; a) negative, b) positive.

Figure 3

Model performance evaluation techniques; a) hold-out b) 5-fold cross-validation

a)

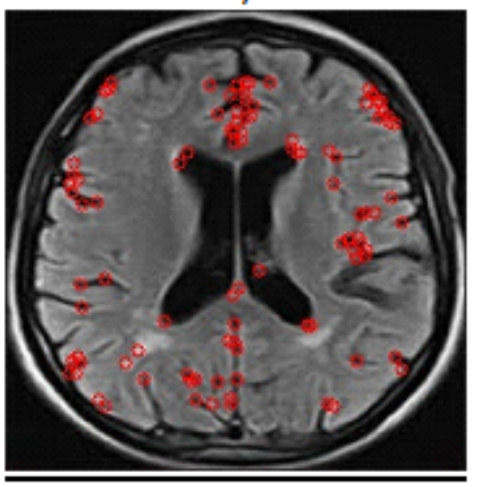

b)

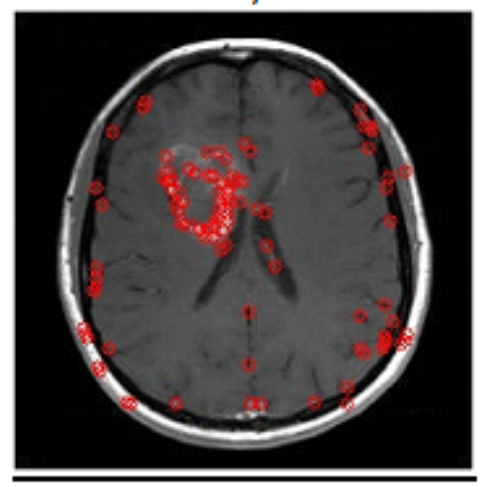




\section{Figure 4}

Keypoints detected on the sample images for each class; From left to right; a) normal case, b) tumour case. (red points are keypoints detected on the image)

Figure 5

Confusion matrices of the classifiers on the VGG16 deep features with hold-out technique

Figure 6

Confusion matrices of the classifiers on the hypercolumn deep features with hold-out technique

\section{Figure 7}

ROC results with hold-out technique; a) VGG16 deep features, b) Hypercolumn deep features

\section{Figure 8}

Confusion matrices of the classifiers on the hypercolumn deep features with 5-fold cross-validation technique

\section{Figure 9}

ROC results for hypercolumn features with 5 -fold cross-validation technique 
Figure 10

FNRs of the classifiers

Figure 11

Classification results of the models 